\title{
Efficacy of a Combination of Tanacetum parthenium, 5-Hydroxy Tryptophan and Magnesium (Aurastop $($ ) ) in Episodic Migraine Prevention: A Multicentric Observational Study
}

\author{
Federico Mainardi' ${ }^{\text {, Paola Merlo }}{ }^{2}$, Ferdinando Maggioni ${ }^{3}$, Giorgio Zanchin ${ }^{3}$, Giorgio Dalla Volta ${ }^{4}$ \\ ${ }^{1}$ Headache Centre, Neurological Division, SS Giovanni e Paolo Hospital, Venice, Italy \\ ${ }^{2}$ Headache Centre of Neurological Division of Gavazzeni Hospital, Bergamo, Italy \\ ${ }^{3}$ Headache Centre, Department of Neurosciences, Padua University, Padua, Italy \\ ${ }^{4}$ Headache Center of Neurological Unit of Istituto Clinico Città di Brescia, Brescia, Italy \\ Email: ^dalla@numerica.it
}

How to cite this paper: Mainardi, F., Merlo, P., Maggioni, F., Zanchin, G. and Volta, G.D. (2018) Efficacy of a Combination of Tanacetum parthenium, 5-Hydroxy Tryptophan and Magnesium (Aurastop $\odot$ ) in Episodic Migraine Prevention: A Multicentric Observational Study. Open Access Library Journal, 5: e4765.

https://doi.org/10.4236/oalib.1104765

Received: July 9, 2018

Accepted: August 3, 2018

Published: August 6, 2018

Copyright $\odot 2018$ by authors and Open Access Library Inc.

This work is licensed under the Creative Commons Attribution International License (CC BY 4.0).

http://creativecommons.org/licenses/by/4.0/

\section{(c) (i) Open Access}

\begin{abstract}
Objective: To verify the efficacy and safety of the new combination of Tanacetum parthenium $150 \mathrm{mg}$, 5-hydrossitriptophan (5-HTTP) $20 \mathrm{mg}$ and magnesium $185 \mathrm{mg}$ (Aurastop ${ }^{\oplus}$ ) in the prophylactic treatment of episodic migraine without aura (MO). Methods: Eighty patients suffering from MO for at least 6 months with a monthly frequency of 3 to 8 attacks and 4 to12 headache days, were enrolled in this open study and treated with Aurastop twice daily per os for 3 months. The reduction of headache days per month was assessed as the primary end-point, while the secondary end-points were: 1) reduction of the number of MO attacks; 2) reduction of intensity of the pain; 3 ) reduction of acute treatment drug intake; 4) subjective change of pain intensity. Results: All the parameters significantly improved at the end of the observational period of treatment with Aurastop. In more detail: a significant reduction of the number of headache days (from $9.1 \pm 2.0$ before treatment to $3.2 \pm 1.8$ post treatment, $\mathrm{p}<0.001$ ); number of attacks per month (from $6.0 \pm$ 1.2 to $2.4 \pm 1.1, \mathrm{p}<0.001$ ); pain intensity (in a visual analogical scale [VAS]: from $7 \pm 1.0$ to $3.2 \pm 0.7, \mathrm{p}<0.001$ ); number of drug doses for acute treatment (triptans, simple analgesics or in combination) assumed by each subject per month (from $9.5 \pm 1.8$ to $2.2 \pm 1.1, \mathrm{p}<0.001$ ). No serious adverse events were observed. Conclusion: Albeit obtained with the limitation of open-trials, our findings suggest that AURASTOP ${ }^{\circledast}$ is a promising approach for migraine prevention; further investigations are required to confirm the safety and efficacy of this treatment.
\end{abstract}




\section{Subject Areas}

Neurology

\section{Keywords}

Migraine without Aura, Kynurenine Pathway, Tanacetum parthenium

\section{Introduction}

Neurological disorders are considered as the principal cause group of disability-adjusted life years; among them, migraine ranked as the second most frequent condition [1]; epidemiologic studies have consistently indicated its prevalence being up to $18 \%$ among women and $6 \%$ among men in Western Countries, with the peak reached between 22 and 55 years [2], and a high impact in terms of social and economic costs [3]. In Italy, the direct and indirect costs of migraine have been estimated to be about 6 billion Euros per year.

To reduce the global impact of migraine, it is generally accepted that a prophylactic treatment should be started in patients who present disabling migraine attacks for at least 4 days per month, and/or in the case of inefficacy of appropriate symptomatic approaches.

The main purpose of migraine prophylaxis is to reduce the frequency of attacks, the number of headache day per month, the patient disability, and consequently to improve the quality of life. A prophylactic therapy is considered effective when it reduces the frequency of migraine attacks of at least $50 \%$.

According to national and international therapeutic guidelines, first-line drugs for migraine prophylaxis include beta-blockers, calcium antagonists, anti-depressants, anti-epileptics, onabotulinumtoxinA and phytotherapics plus magnesium [4].

However, a number of migraineurs are unsatisfied from the standard prophylactic drugs because of the lack of efficacy and/or side effects; in these cases, nutraceuticals could be proposed for migraine prevention.

The term "nutraceuticap", a blend of the words "nutrition" and "pharmaceutical", was coined by DeFelice in 1989 and indicates a food or a part of food that has beneficial health effects and could be used in the prevention or treatment of a disease.

In the last years the use of nutraceuticals products by migraine has increased as reported by the National Health Interview study [5]; according to the results of this survey, $49.5 \%$ of the patients use nutraceutical products without informing their health care providers.

Recently, Scientific Societies have reviewed the principal studies published on the topic with the aim to update the therapeutical guidelines and, if the case, insert nutraceuticals for migraine prevention according to their level of evidence [6]. 


\section{Material and Methods}

Eighty patients with episodic migraine as defined by the International Classification of Headache Disorders 3 beta (ICHD 3 beta) [7] were consecutively recruited from January 2017 to the end of the year, among those referring to the Headache Centres of the Istituto Clinico Citta' di Brescia (Brescia), Gavazzeni Hospital (Bergamo) and San Giovanni e Paolo Hospital (Venice). The approval of the local Ethic Committee had been obtained. (Np 2465 of 4th Jan 2017-Brescia).

Criteria for patients selection were: 1 ) age between 18 and 65 years; 2) diagnosis of MO, according to the ICHD 3 beta diagnostic criteria; 3 ) migraine for at least 6 months with a monthly crisis frequency ranging from 3 to $10 ; 4$ ) headache duration ranging from 4 to 12 days per month. Psychiatric co-morbidity, other types of acute or chronic pain, kidney failure, neurological or oncologic diseases, and pregnancy were considered as exclusion criteria.

A preventative treatment had been started before the beginning of the survey and was continued during the study in 5 cases (propranolol $40 \mathrm{mg}$ tablet bid since 2 months: $\mathrm{n}=2$; amitriptyline 12 drops since 1 month: $\mathrm{n}=2$; onabotulinumtoxinA: 1 session of 31 injection as for PREEMPT study $n=1$ ).

At baseline evaluation all patients underwent a thorough a neurological examination and were carefully instructed on how to record MO attacks in their headache diary on a day-to-day basis. In particular, during the observational period of three months they were asked to register: 1) attacks frequency; 2) pain intensity (using the VAS scale, ranging from 1 to 10 ); 3) duration; 4) response to analgesics, based on a self-rate scale ranging between 1 (minimum response) and 5 (maximum response). At the end of the screening phase, diary cards were controlled and the eligible patients received Aurastop ${ }^{\oplus}$ (a combination of Tanacetum parthenium $150 \mathrm{mg}$, 5-hydrossitriptophan (5-HTTP) $20 \mathrm{mg}$ and magnesium $185 \mathrm{mg}$ ) twice daily for 3 months. Safety was evaluated by treatment discontinuation rate and the occurrence of serious and otherwise adverse events.

The primary endpoint of the study was the reduction of headache days per month. The secondary endpoints were: 1 ) reduction of MO attacks; 2) reduction of intensity of the pain; 3) reduction of acute treatment drugs intake; 4) subjective change of pain intensity.

\section{Statistical Analysis}

Categorical variables are reported as counts and percentages. Dependent variables were compared by McNemar's $\chi^{2}$ analysis. Wilcoxon's signed rank test was used to compare migraine characteristics before and after treatment. Statistical analyses were performed using SPSS 21.0 (IBM SPSS Statistics 2013, Armonk, NY, USA).

\section{Results}

Overall, 80 patients ( 35 males, 45 females; mean age: 37.5 years, range 19 - 60) 
were enrolled in the study. One patient withdrew after 1 month of treatment because of lack of improvement and one was lost to follow-up.

The results of the analysis are summarized in Table 1 . that show a significant reduction of the number of headache days (from $9.1 \pm 2.0$ before treatment to $3.2 \pm 1.8$ post treatment, $\mathrm{p}<0.001$ ), as well as of the number of attacks per month (from $6.0 \pm 1.2$ to $2.4 \pm 1.1, \mathrm{p}<0.001$ ), pain intensity (in a visual analogical scale [VAS]: from $7 \pm 1.0$ to $3.2 \pm 0.7, \mathrm{p}<0.001$ ), and number of drug doses for acute treatment (triptans, simple analgesics or in combination) assumed by each subject (from $9.5 \pm 1.8$ to $2.2 \pm 1.1, \mathrm{p}<0.001$ ) (Figure 1 ). None of the patients reported any adverse events related to the treatment.

Another notable finding derived from the analysis of the headache diaries, was that the results at the 3-month time point reported above were already detectable after one month of prophylactic therapy with Aurastop.

\section{Discussion}

The mechanisms of action of nutraceuticals in migraine prevention could be hypothesised on the basis of pre-clinical observations, that demonstrated their effects on the vessel wall, neurons, and specific neurotransmitters involved in the pathophysiology of migraine. Albeit evidences suggest the role of the cerebrovascular system as well as an abnormal cortical activity and immunological involvement, the exact pathophysiology of migraine remains not completely

Table 1. Migraine characteristics before and after prophylactic treatment with Aurastop.

\begin{tabular}{cccc}
\hline Parameter & Baseline & Post-treatment & p-value \\
\hline Number of days per month & $9.1 \pm 2.0$ & $3.2 \pm 1.8$ & $\mathrm{p}<0.001$ \\
Number of attacks per month & $6.0 \pm 1.2$ & $2.4 \pm 1.1$ & $\mathrm{p}<0.001$ \\
Pain intensity (VAS: 0 - 10) & $7 \pm 1.0$ & $3.2 \pm 0.7$ & $\mathrm{p}<0.001$ \\
Number of analgesics per month & $9.5 \pm 1.8$ & $2.2 \pm 1.1$ & $\mathrm{p}<0.001$ \\
\hline
\end{tabular}

$\square$ Baseline $\square$ Post-treatment

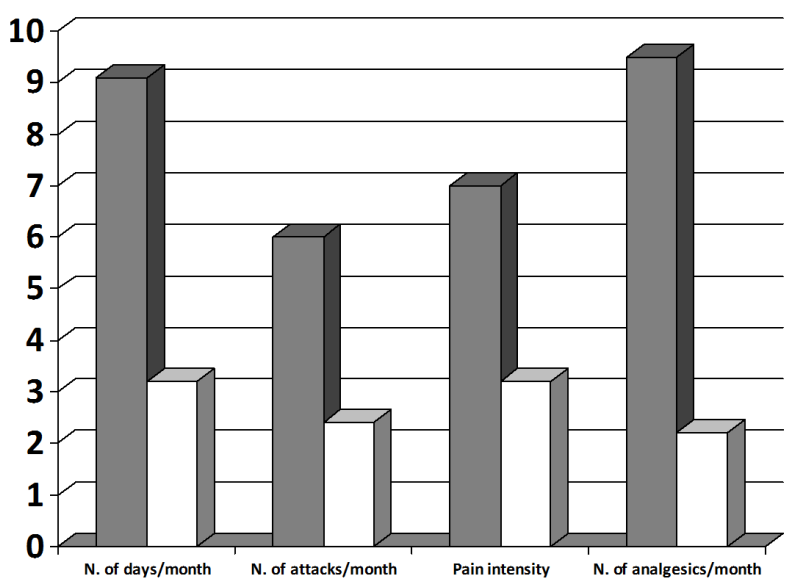

Figure 1. Primary and secondary end-points. 
understood and a multifactorial origin has been proposed.

Whatever the exact mechanism, the central sensitization of the sensory neurons innervating intracranial meninges and their related large blood vessels appears to be accountable for the persistence of pain and its throbbing quality. Sensitization results from the local release of neuropeptides that cause vasodilatation and an increase in vascular permeability, respectively mediated by calcitonin gene-related peptide and substance $P$, neurokinin $A$ and mast cells activation.

Moreover, a sterile meningeal inflammatory response is widely considered as an adjunctive factor that lead to the central sensitization of primary afferent meningeal nociceptive neurons, apparently one of the earliest events promoting the onset of pain. In this process, the activation of resident mast cells is a critical step in promoting an enhanced excitability of meningeal nociceptors, by releasing pro-inflammatory/algesic mediators that induce vasodilatation and are responsible of the throbbing quality of pain. As a consequence, the inhibition of the activity of the mast cells could result in a reduction of the excitability of the meningeal nociceptors.

Parthenolide could modulate mast cells activation by the inhibition of the I $\mathrm{B}$ kinase complex (IKK) $\beta$, which is involved in the proinflammatory cytokine-mediated signaling. Furthermore, parthenolide interacts with the TRPA1 nucleophilic sites. The antimigraine effect of parthenolide could derive both from its partial agonism on TRPA1 and the inhibition of CGRP release obtained by desensitization and nociceptor defunctionalization [5] [6] [8]. Glutamate is another neuropeptide likely involved in migraine pathogenesis due to its excitatory effect on first and second order neurons and its role in the activation of the trigeminovascular system. Moreover, the post-synaptic glutamatergic receptor N-methyl-D-Aspartate (NMDA) is involved on the occurrence of both central sensitization and cortical spreading depression, as demonstrated by its activation during migraine attacks [9]. NMDA receptors are activated by an increase of the synaptic levels of glutamate and inhibited by magnesium [10] [11] [12].

Glutamate levels are regulated by kynurenine [13] which metabolizes 1-triptophan in kynurenic acid (KYNA) and quinolinic acid (QUINA). In particular, the NMDA receptor antagonist KYNA inhibits glutamatergic pathway by blocking glutamate release and neurotransmission through its action on the binding site of glycin Glu N1. Recently, it has been observed that in migraineurs the kynuretic pathway is shifted towards the conversion of KYNA in antralinic acid (ANA); this observation is supported by the finding of elevated plasma levels of ANA, which has also a direct neurotoxic effect through the release of free radicals, and reduced concentration of KYNA and QUINA, leading to a subsequent loss of inhibitory control on glutamate and its excitatory effects.

Low plasma levels of KYNA may be considered a reliable marker of NMDA receptor activation, while its cerebral levels can be increased by the assumption of its precursor 5-HTP [14]. If used as a drug, this latter may, therefore, increase KYNA levels inhibit peripheral NMDA receptors and subsequently prevent the activation of the trigeminovascular system and the onset of CSD. 
In addition, TRPA1 and NMDA receptors, glutamate and calcitonin-gene-related peptide (CGRP) are involved in the neurogenic inflammation process, which leads to the sensitization of trigeminal nucleus caudalis in the lower brainstem and upper cervical cord and, consequently, of all the structures implicated in the central transmission of nociceptive information.

Molecules that could trigger a migraine attack act as agonist on TRP receptors, leading to a neurogenic inflammation through the release of CGRP from perivascular nerve terminals. Such a cascade might be interrupted by parthenolide, a TRP receptors inhibitor. Moreover, parthenolide is a powerful inhibitor of nitric oxide (NO) synthase and, consequently, of NO production. Moreover, among its many actions, intracellular magnesium has a physiologic calcium-antagonist effect, resulting in a reduction of the toxic effects of calcium; on the contrary, suboptimal concentrations of this ion favor a free radical accumulation within the cell, which, in turn, may facilitate the onset of a migraine attack. Furthermore, magnesium inhibits NMDA receptors, which are involved in the glutamate-dependent excitatory pathways at the basis of the neurogenic inflammation.

Based on the mechanisms described above, the combination of the three components, Tanacetum parthenium, 5-HTP, and magnesium is expected to synergistically influence the biologic pathways involved in migraine pathogenesis, and, therefore, to have a therapeutic potential in migraine prevention.

The results of the present study highlight the efficacy and the safety profile of the combination of Tanacetum parthenium, 5-HTP and magnesium in MO prophylaxis. Both the frequency and duration of migraine attacks as well as the intensity of the pain significantly improved in most of the patients. These findings, in addition to the more favorable response to analgesics and the reduced number of the consumption of these medications in patients receiving Aurastop, lead to the speculation that the combination of the three molecules might act synergistically on the different pathways involved in migraine pathophysiology, that is: neurogenic inflammation, neural transmission, and central sensitization. CSD, involved in the pathogenesis of both migraine without and with aura, has been consistently related to the status of inter-critical cortical brain hyperexcitability of migraineurs.

Therefore, all the findings observed in the present study can be interpreted as the clinical manifestation of Aurastop ${ }^{\otimes}$ action on central sensitization, central transmission of nociceptive information at synaptic level, TRPA1 channels, and NMDA receptors.

The results of the present study highlight the efficacy and the safe profile of the combination of Tanacetum parthenium, 5-HTP and magnesium in migraine prophylaxis.

Both the primary endpoint represented by the reduction of the headache days a month and the secondary endpoints represented by the reduction of the number of attacks, intensity of the pain as well as the number of analgesics assumed, 
improved significantly in a significantly percentage of the patients treated.

These findings are most similar to that emerged from a similar previous study of Merlo, P. [15], even if with a smaller group of patients, and of the first study using Aurastop of Zavarise, P. [16] to testify the efficacy of this combination of molecules.

These findings lead to the speculation that the 3 molecules in combination might act synergistically on different pathways involved in migraine biology: neurogenic inflammation, neural transmission, and central sensitization. The well-known phenomenon of cortical spreading depression (CSD), involved in the pathogenesis of both migraine without aura and migraine with aura, has been consistently related to the status of inter-critical cortical brain hyperexcitability of migraineurs. This emphasizes the physiologic role of magnesium in the regulation of such a neuronal excitability. Among the many actions, intracellular magnesium acts as a physiologic calcium-antagonist, thus reducing the toxic effects of calcium, whereas suboptimal concentrations of magnesium favor free radical accumulation within the cell, which, in turn, may trigger a migraine attack. Furthermore, magnesium inhibits NMDA receptors, which are involved in the glutamate-dependent excitatory pathways at the basis of the neurogenic inflammation. Moreover, brain concentration of kynurenic acid (Kyna), a tryptophan derivative which further acts as an endogenous NMDA receptor antagonist, increases with increasing serum levels of its precursor 5HTP. Giving 5HTP as a drug may, therefore, increase Kyna levels and influence Kyna pathways, leading to the inhibition of peripheral NMDA receptors and the consequent activation of the trigeminovascular system, as well as of CSD.

Finally, TRPA1 and NMDA receptors with glutamate and calcitonin-gene-related peptide (CGRP) play a key role in neurogenic inflammation, which leads to the sensitization of trigeminal nucleus caudalis in the lower brainstem and upper cervical cord and, consequently, of all structures involved in the central transmission of nociceptive information.

Molecules proved to be migraine generators act as TRP receptors activators. These, in turn, generate neurogenic inflammation leading to painful attacks through the release of CGRP from perivascular nerve terminals. Such a cascade might be interrupted by parthenolide, a TRP receptors inhibitor. Parthenolide is, moreover, a powerful inhibitor of nitric oxide synthase and, consequently, of nitric oxide (NO) production. All the findings observed in the present study can, therefore, be interpreted as clinical manifestation of the interference with the phenomenon of central sensitization, as well as with the central transmission of nociceptive information at synaptic level, TRPA1 channels, and NMDA receptors determined by Aurastop.

\section{Conclusion}

In spite of the methodological limitations of this observational pilot study due to the absence of a placebo group, our findings emphasize the potential effect of 
Aurastop ${ }^{\otimes}$ on the complex physiopathological mechanisms of migraine and confirmed our previous observation [15]. The combination Tanacetum parthenium + 5-HTP + magnesium appears to be a promising alternative therapy for migraine prophylaxis with an excellent safety profile. Further clinical trials are needed in order to investigate its potentials in preventing migraine, either alone or in combination with other molecules.

\section{Conflicts of Interest}

The authors declare no conflicts of interest regarding the publication of this paper.

\section{References}

[1] GBD 2015 Neurological Disorder Collaborator Group (2017) Global, Regional and National Burden of Neurological Disorders during 1990-2015; A Systematic Analysis for the Global Burden of Disease Study 2015. Lancet Neurology, 16, 877-897.

[2] Lipton, R.B., Bigal, M.E., Diamond, M., et al. (2007) Migraine Prevalence, Disease Burden, and the Need for Preventive Therapy. Neurology, 68, 343-349.

https://doi.org/10.1212/01.wnl.0000252808.97649.21

[3] Bloudek, L.M., Stokes, M., Buse, D.C., Wilcox, T.K., Lipton, R.B., Goadsby, P.J., et al. (2012) Cost of Healthcare for Patients with Migraine in Five European Countries: Results from the International Burden of Migraine Study (IBMS). Journal of Headache and Pain, 13, 361-378. https://doi.org/10.1007/s10194-012-0460-7

[4] Rajapakse, T. and Pringsheim, T. (2016) Nutraceuticals in Migraine: A Summary of Existing Guidelines for Use. Headache, 56, 808-816.

https://doi.org/10.1111/head.12789

[5] Diener, H.C., Pfaffenrath, V., Schnitker, J., Friede, M. and Henneicke-von Zepelin, H.H. (2005) Efficacy and Safety of $6.25 \mathrm{mg}$ t.i.d. Feverfew CO2-Extract (MIG-99) in Migraine Prevention-A Randomized, Double-Blind, Multicentre, Placebo-Controlled Study. Cephalalgia, 25, 1031-1041. https://doi.org/10.1111/j.1468-2982.2005.00950.x

[6] Tassorelli, C., Greco, R., Morazzoni, P., Riva, A., Sandrini, G. and Nappi, G. (2005) Parthenolide Is the Component of Tanacetum parthenium That Inhibits Nitroglycerin-Induced Fos Activation: Studies in an Animal Model of Migraine. Cephalalgia, 25, 612-621. https://doi.org/10.1111/j.1468-2982.2005.00915.x

[7] Headache Classification Committee of the International Headache Society (2013) The International Classification of Headache Disorders, 3rd Edition (Beta Version). Cephalalgia, 33, 629-808.

[8] Materazzi, S., Benemei, S., Fusi, C., et al. (2013) Parthenolide Inhibits Nociception and Neurogenic Vasodilatation in the Trigeminovascular System by Targeting the TRPA1 Channel. Pain, 154, 2750-2758. https://doi.org/10.1016/j.pain.2013.08.002

[9] Begon, S., Pickering, G., Eschalier, A., et al. (2001) Role of Spinal NMDA Receptors, Protein Kinase $C$ and Nitric Oxide Synthase in the Hyperalgesia Induced by Magnesium Deficiency in Rats. British Journal of Pharmacology, 134, 1227-1236. https://doi.org/10.1038/sj.bjp.0704354

[10] Sun-Edelstein, C. and Mauskop, A. (2009) Role of Magnesium in the Pathogenesis and Treatment of Migraine. Expert Review of Neurotherapeutics, 9, 369-379. https://doi.org/10.1586/14737175.9.3.369

[11] Mody, I., Lambert, J.D. and Heinemann, U. (1987) Low Extracellular Magnesium 
Induces Epileptiform Activity and Spreading Depression in Rat Hippocampal Slices. Journal of Neurophysiology, 57, 869-888. https://doi.org/10.1152/jn.1987.57.3.869

[12] Teigen, L. and Boes, C.J. (2015) An Evidence-Based Review of Oral Magnesium Supplementation in the Preventive Treatment of Migraine. Cephalalgia, 35, 912-922. https://doi.org/10.1177/0333102414564891

[13] Chauvel, V., Vamos, E., Pardutz, A., et al. (2012) Effect of Systemic Kynurenine on Cortical Spreading Depression and Its Modulation by Sex Hormones in Rat. Experimental Neurology, 236, 207-214.

https://doi.org/10.1016/j.expneurol.2012.05.002

[14] Curto, M., Lionetto, L., Negro, A., et al. (2015) Altered Kynurenine Pathway Metabolites in Serum of Chronic Migraine Patients. Journal of Headache and Pain, 17, 47. https://doi.org/10.1186/s10194-016-0638-5

[15] Merlo, P., Maggioni, F., Zanchin, G., et al. (2017) Efficacy in Episodic Migraine Prevention of a Combination of Tanacethum parthenium, 5-Hydroxy Tryptophan and Magnesium (Aurastop $\odot$ ). A Multicentric Observational Study. Journal of Headache and Pain, 18, 202.

[16] Zavarize, P., Pezzini, A. and Dalla Volta, G. (2017) Combination of Tanacethum partenium, 5-Hydrossitriptophan (5-Http) and Magnesium in the Prophylaxis of Episodic Migraine without Aura (AURASTOP ${ }^{\circledast}$ ) An Observational Study. International Journal of Neurology and Brain Disorders, 4, 1-4.

https://doi.org/10.15436/2377-1348.17.1630 